\title{
HYPERGEOMETRIC SERIES ACCELERATION VIA THE WZ METHOD
}

\author{
Tewodros Amdeberhan and Doron Zeilberger \\ Department of Mathematics, Temple University, Philadelphia PA 19122, USA \\ tewodros@math.temple.edu, zeilberg@math.temple.edu
}

Submitted: Sept 5, 1996. Accepted: Sept 12, 1996

Dedicated to Herb Wilf on his one million-first birthday

\begin{abstract}
Based on the WZ method, some series acceleration formulas are given. These formulas allow us to write down an infinite family of parametrized identities from any given identity of WZ type. Further, this family, in the case of the Zeta function, gives rise to many accelerated expressions for $\zeta(3)$.
\end{abstract}

AMS Subject Classification: Primary 05A

We recall[ [Z] that a discrete function $\mathrm{A}(\mathrm{n}, \mathrm{k})$ is called Hypergeometric (or Closed Form $(\mathrm{CF})$ ) in two variables when the ratios $A(n+1, k) / A(n, k)$ and $A(n, k+1) / A(n, k)$ are both rational functions. A discrete 1-form $\omega=F(n, k) \delta k+G(n, k) \delta n$ is a $W Z 1$-form if the pair (F,G) of CF functions satisfies $F(n+1, k)-F(n, k)=G(n, k+1)-G(n, k)$.

We use: $\mathrm{N}$ and $\mathrm{K}$ for the forward shift operators on $\mathrm{n}$ and $\mathrm{k}$, respectively. $\Delta_{n}:=N-1, \Delta_{k}:=K-1$.

Consider the WZ 1-form $\omega=F(n, k) \delta k+G(n, k) \delta n$. Then, we define the sequence $\omega_{s}, s=1,2,3, \ldots$ of new WZ 1-forms: $\omega_{s}:=F_{s} \delta k+G_{s} \delta n$; where

$$
F_{s}(n, k)=F(s n, k) \quad \text { and } \quad G_{s}(n, k)=\sum_{i=0}^{s-1} G(s n+i, k) .
$$

Proposition: $\omega_{s}$ is WZ, for all s.

Proof: (a) $\omega_{s}$ is closed:

$$
\begin{aligned}
\Delta_{n} F_{s} & =F(s(n+1), k)-F(s n, k) \\
& =\sum_{i=0}^{s-1}(F(s n+i+1, k)-F(s n+i, k)) \\
& =\sum_{i=0}^{s-1}(G(s n+i, k+1)-G(s n+i, k)) \\
& =\sum_{i=0}^{s-1} G(s n+i, k+1)-\sum_{i=0}^{s-1} G(s n+i, k) \\
& =\Delta_{k} G_{s} .
\end{aligned}
$$


Note that since $\omega$ is a WZ, it has the form [Z], p.590):

$$
\omega=f(n, k)(P(n, k) \delta k+Q(n, k) \delta n)
$$

for some $\mathrm{CF}$ f and some polynomials $\mathrm{P}$ and $\mathrm{Q}$.

(b) $\omega_{s}$ has the form $(*)$ :

Indeed, $\omega_{s}$ can be rewritten as:

$$
\begin{aligned}
\omega_{s} & =f(s n, k)\left(P(s n, k) \delta k+\sum_{i=0}^{s-1} \frac{f(s n+i, k)}{f(s n, k)} Q(s n+i, k) \delta n\right) \\
& =f(s n, k)(P(s n, k) \delta k+R(n, k) \delta n) ;
\end{aligned}
$$

where $R(n, k)$ is a rational function and $f(s n, k)$ is still CF. Hence after pulling out a common denominator, we see that $\omega_{s}$ too has the form $(*)$. This proves the Proposition.

Theorem 1: $[(\mathrm{Z}]$, Theorem 7, p.596) For any WZ pair $(\mathrm{F}, \mathrm{G})$

$$
\sum_{n=0}^{\infty} G(n, 0)=\sum_{n=1}^{\infty}(F(n, n-1)+G(n-1, n-1))-\lim _{n \rightarrow \infty} \sum_{k=0}^{n-1} F(n, k)
$$

whenever both side converge.

Formula 1:

$$
\sum_{n=0}^{\infty} G(n, 0)=\sum_{n=0}^{\infty}\left(F(s(n+1), n)+\sum_{i=0}^{s-1} G(s n+i, n)\right)-\lim _{n \rightarrow \infty} \sum_{k=0}^{n-1} F(s n, k) .
$$

Proof: Apply Theorem 1 above on $\omega_{s}$. Alternatively, integrate $\omega$ along the boundary contour $\partial \Omega_{s}$ of the region $\Omega_{s}=\{(n, k): s n \geq k\}$.

Formula 2: We also have that

$$
\sum_{k=0}^{\infty} F(0, k)-\lim _{n \rightarrow \infty} \sum_{k=0}^{n} F(n, k)=\sum_{n=0}^{\infty} G(n, 0)-\lim _{k \rightarrow \infty} \sum_{n=0}^{k} G(n, k),
$$

whenever both side converge.

Proof: Integrate $\omega$ along the boundary contour $\partial \Omega_{0}$ of the region $\Omega_{0}=\{(n, k): n \geq 0, k \geq 0\}$. 
Remark: By shear symmetry, a formulation similar to (2) can be given in ' $k$ '. And a combination leads to:

Formula 3: For $\omega_{s, t}=F_{s, t} \delta k+G_{s, t} \delta n$; where

$$
\begin{aligned}
& F_{s, t}(n, k)=\sum_{j=0}^{t-1} F(s n, t k+j) \quad \text { and } \quad G_{s, t}(n, k)=\sum_{i=0}^{s-1} G(s n+i, t k), \quad \text { we have } \\
& \sum_{n=0}^{\infty} G(n, 0)=\sum_{n=0}^{\infty}\left(\sum_{j=0}^{t-1} F(s(n+1), t n+j)+\sum_{i=0}^{s-1} G(s n+i, t n)\right)-\lim _{n \rightarrow \infty} \sum_{k=0}^{n-1} F_{s, t}(n, k) .
\end{aligned}
$$

Analogous statements hold in several variables. To wit:

for the WZ 1-form in 3 variables, $\omega_{s, t, r}:=F_{s, t, r} \delta k+G_{s, t, r} \delta n+H_{s, t, r} \delta a$; where

$$
\begin{aligned}
F_{s, t, r}(n, k, a) & =\sum_{j=0}^{t-1} F(s n, t k+j, r a), \quad G_{s, t, r}(n, k, a)=\sum_{i=0}^{s-1} G(s n+i, t k, r a) \quad \text { and } \\
H_{s, t, r}(n, k, a) & =\sum_{u=0}^{r-1} H(s n, t k, r a+u),
\end{aligned}
$$

\section{Formula 4:}

$$
\begin{aligned}
\sum_{n=0}^{\infty} H(0,0, n)=\sum_{n=0}^{\infty}( & \left.\sum_{u=0}^{r-1} H(s(n+1), t(n+1), r n+u)+\sum_{j=0}^{t-1} F(s(n+1), t n+j, r n)+\sum_{i=0}^{s-1} G(s n+i, t n, r n)\right) \\
& -\lim _{a \rightarrow \infty} \sum_{k=0}^{a+1} F_{s, t, r}(a+1, k, a)-\lim _{a \rightarrow \infty} \sum_{n=0}^{a+1} G_{s, t, r}(n, a+1, a) .
\end{aligned}
$$

In [A] formula (1) was used to give a list of series acceleration for $\zeta(3)$ (where $F(n, k)$ is given and its companion $\mathrm{G}(\mathrm{n}, \mathrm{k})$ is produced by the amazing Maple Package EKHAD accompanying [PWZ]). A small Maple Package accel applying (3) is available at http://www.math.temple.edu/ [tewodros, zeilberg].

For example: with $F(n, k)=(-1)^{k} \frac{n !^{6}(2 n-k-1) ! !^{3}}{2(n+k+1) ! 2(2 n) !^{3}}, \mathrm{~s}=1$ and $\mathrm{t}=1$ accel produces the following pretty formula:

$$
\zeta(3)=\sum_{n=0}^{\infty}(-1)^{n} \frac{n !^{10}\left(205 n^{2}+250 n+77\right)}{64(2 n+1) !^{5}} .
$$

Greg Fee and Simon Plouffe used $(* *)$ in their evaluation of $\zeta(3)$ to 520,000 digits (available at http://www.cecm.sfu.ca/projects/ISC/records.html).

ACKNOWLEDGMENT: We would like to express our gratitude to Professor Herbert Wilf for his valuable comments and suggestions. 


\section{REFERENCES}

[A] T. Amdeberhan, Faster and faster convergent series for $\zeta(3)$, Elect. Jour. Combin. 3 (1996).

[PWZ] M. Petkovšek, H.S. Wilf, D. Zeilberger, " $A=B$ ", A.K. Peters Ltd., 1996.

The package EKHAD is available by the www at http://www.math.temple.edu/ ${ }^{\sim}$ zeilberg/programs.html

[WZ1] H.S. Wilf, D. Zeilberger, Rational functions certify combinatorial identities, Jour. Amer. Math. Soc. 3 (1990), 147-158.

[Z] D. Zeilberger, Closed Form (pun intended!), Contemporary Mathematics 143 (1993), 579-607. 